\title{
Distinct In Vitro Properties of Embryonic and Extraembryonic Fibroblast-Like Cells Are Reflected in Their In Vivo Behavior Following Grafting in the Adult Mouse Brain
}

\author{
Roberta Costa,* Irene Bergwerf, $\dagger \neq$ Eva Santermans, $\S$ Nathalie De Vocht, $\dagger+$ Jelle Praet,,$\uparrow$ Jasmijn Daans, $\dagger \neq$

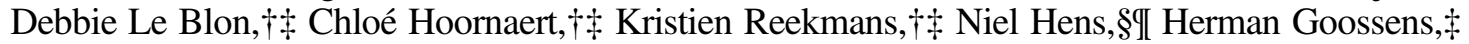 \\ Zwi Berneman, $\dagger \neq$ Ornella Parolini,\# Francesco Alviano,* and Peter Ponsaerts $\dagger \neq$ \\ *Department of Experimental, Diagnostic and Specialty Medicine, University of Bologna, Bologna, Italy \\ $†$ Experimental Cell Transplantation Group, Laboratory of Experimental Hematology, University of Antwerp, Antwerp, Belgium \\ $\$$ Vaccine and Infectious Disease Institute (Vaxinfectio), University of Antwerp, Antwerp, Belgium \\ $\S$ Center for Statistics, I-Biostat, Hasselt University, Hasselt, Belgium \\ ICentre for Health Economic Research and Modeling Infectious Diseases (Chermid), University of Antwerp, Antwerp, Belgium \\ \#Centro di Ricerca E. Menni, Fondazione Poliambulanza - Istituto Ospedaliero, Brescia, Italy
}

\begin{abstract}
Although intracerebral transplantation of various fibroblast(-like) cell populations has been shown feasible, little is known about the actual in vivo remodeling of these cellular grafts and their environment. In this study, we aimed to compare the in vitro and in vivo behavior of two phenotypically similar - but developmentally distinctfibroblast-like cell populations, namely, mouse embryonic fibroblasts (mEFs) and mouse fetal membrane-derived stromal cells (mFMSCs). While both mEFs and mFMSCs are readily able to reduce TNF- $\alpha$ secretion by LPS/IFN- $\gamma$ activated BV-2 microglia, mFMSCs and mEFs display strikingly opposite behavior with regard to VEGF production under normal and inflammatory conditions. Whereas mFMSCs downregulate VEGF production upon coculture with LPS/IFN- $\gamma$-activated BV-2 microglia, mEFs upregulate VEGF production in the presence of LPS/ IFN- $\gamma$-activated BV-2 microglia. Subsequently, in vivo grafting of mFMSCs and mEFs revealed no difference in microglial and astroglial responses toward the cellular grafts. However, mFMSC grafts displayed a lower degree of neoangiogenesis compared to $\mathrm{mEF}$ grafts, thereby potentially explaining the lower cell number able to survive in mFMSC grafts. In summary, our results suggest that physiological differences between fibroblast-like cell populations might lie at the basis of variations in histopathological and/or clinical outcome following cell grafting in mouse brain.
\end{abstract}

Key words: Fetal membrane-derived stromal cells; Embryonic fibroblasts; Immunomodulation; Transplantation; Brain; Angiogenesis

\section{INTRODUCTION}

Transplantation of fibroblastic cells, including adult/ embryonic fibroblasts and mesenchymal stromal cells, is widely being applied in various preclinical animal models of human disease. Major interest into these cell populations can be explained by the relatively easy in vitro isolation (and/or expansion) protocols and the exceptional immune-modulating and regeneration-inducing properties of these cells (2). However, while extensive in vitro experiments have shed light on potential working mechanisms for the improved clinical outcome following fibroblastic cell grafting in several disease models, including those of the central nervous system (CNS), currently little is known about the actual in vivo behavior of these cellular grafts $(1,7)$. In our preceding studies, we contributed to a better understanding of the cellular events following syngeneic reporter gene-modified murine bone marrowderived mesenchymal stromal cell (mBMMSC) and murine embryonic fibroblast $(\mathrm{mEF})$ grafting in the healthy and injured CNS of immune-competent mice $(4,5,10,21)$. In the course of these studies, it was noted that both mMSC and $\mathrm{mEF}$ grafting in the CNS, independent of a preceding injury, results in the activation of strong microglial and astroglial cell responses. Without manifest differences, both mMSC and mEF grafts become highly surrounded and infiltrated with ionized calcium-binding adapter molecule

Received September 3, 2013; final acceptance December 3, 2013. Online prepub date: December 30, 2013.

Address correspondence to Prof. Dr. Peter Ponsaerts, Experimental Cell Transplantation Group, Laboratory of Experimental Hematology,

Vaccine and Infectious Disease Institute (Vaxinfectio), University of Antwerp, Campus Drie Eiken (CDE-S6.51), Universiteitsplein 1,

2610 Antwerp (Wilrijk), Belgium. Tel: +32-3-2652428; E-mail: peter.ponsaerts@uantwerpen.be 
1-positive $\left(\mathrm{Iba}^{+}\right)$microglia(/macrophages). In addition, both mMSC and mEF grafts become surrounded, but are not infiltrated, by highly activated glial fibrillary acidic protein-positive $\left(\mathrm{GFAP}^{+}\right)$astrocytes. Given the in vivo occurrence of cellular immune responses against grafted fibroblastic cells, although we do not yet know the exact mechanism behind them, further research toward identifying a cell population that triggers no or minimal CNS immune responses upon transplantation is highly desirable. The latter will not only lead to the application of safer therapeutic cell-based therapies, but, more importantly, might lead to the establishment of therapeutic interventions in which functional cell integration might occur in the adult immune-competent CNS (5).

Recently, the placenta, fetal membranes, and amniotic fluid have been put forward as alternative sources for cells of mesenchymal origin in cell transplantation experiments $(16,29)$. Based on the observation that during pregnancy the competent immune system of the mother is rendered tolerant to the immunologically distinct fetus, the placenta and fetal membranes, which are the interface between mother and fetus, may harbor these immuneregulating properties. The hypothesis that fetal membranes are a nonimmunogenic tissue has been confirmed by several clinical studies that used amniotic membranes for treatment of skin wounds, dermal burns, nonhealing ulcers, ocular surface reconstruction, or to prevent tissue adhesion in surgical procedures $(12,16)$. In these clinical studies, no immune-mediated rejection was observed in the absence of immune-suppressive treatment. Moreover, fibroblastlike cells isolated from fetal membranes, generally termed fetal membrane-derived stromal cells (FMSCs), can present the same immunological behavior. In this context, several preclinical studies in animal models of disease demonstrated that transplantation of human (h)FMSCs significantly contributes to anti-inflammatory and antiscarring processes rather than actual cell replacement. For example, hFMSC transplantation for the treatment of liver and lung fibrosis in rodents showed a reduction in neutrophil infiltration and in the dimension of the fibrotic lesion $(8,17)$. This immunological behavior of hFMSCs has also been observed following neural injury. Transplantation of hFMSCs after stroke in rodents has shown to reduce injury progression by modulating the inflammatory response and facilitating functional recovery $(16,19)$. Furthermore, other in vivo experiments have demonstrated long-term survival of hFMSCs after xenogeneic transplantation into immune-competent animals $(12,14)$.

However, despite demonstrating significant immunomodulating effects, the xenogeneic transplantation of human cells into rodents has significant limitations when aiming to translate the observed clinical benefits to human clinical applications that will be performed with autologous or allogeneic cell grafts. Therefore, in this novel study, we first optimized an isolation procedure for murine (m) FMSCs and characterized the resulting mFMSC population in vitro and in vivo following syngeneic grafting in the CNS. Comparing the obtained data with a mEF control cell population, this study describes functional differences between fibroblast-like cell populations isolated from different tissues, both in vitro and in vivo upon grafting in the CNS.

\section{MATERIALS AND METHODS}

\section{Animals}

Female wild-type C57BL/6 mice (strain code 027; Charles River Laboratories, Chatillon-sur-Chalaronne, France) were crossed with male transgenic C57BL/6-eGFP mice (strain code 003291; Jackson Laboratories, Sacramento, CA, USA) and a total of 29 enhanced green fluorescent protein-positive $\left(\mathrm{eGFP}^{+}\right)$and $71 \mathrm{eGFP}^{-}$embryos/fetal membranes with a mixed gender background were used for cell isolation experiments. Male wild-type C57BL/6 mice (strain code 027; Charles River Laboratories), 8-9 weeks of age, were used for cell implantation experiments $(n=10)$. For all experiments, mice were kept under normal day-night cycle (12-12) with free access to water and food. All experimental procedures were approved by the ethical committee for animal experiments of the University of Antwerp (approval No. 2011-13).

\section{Cell Culture}

mFMSCs were isolated from $\mathrm{eGFP}^{+}$and $\mathrm{eGFP}^{-}$fetal membranes surrounding, respectively, $\mathrm{eGFP}^{+}$and $\mathrm{eGFP}^{-}$ embryos following crossing of female wild-type C57BL/6 mice with male transgenic C57BL/6-eGFP mice. For this, embryos (E17-18) were removed from the uterus, and each fetal membrane was separated manually from the placenta and the embryo. Fetal membranes were recovered, washed with phosphate-buffered saline (PBS; Gibco/Life Technologies Europe, Ghent, Belgium), and enzymatically digested by the following consecutive dissociation steps: $3 \mathrm{~min}$ in PBS supplemented with Dispase (2.5 U/ml, Gibco), $3 \mathrm{~h}$ in Roswell Park Memorial Institute (RPMI) medium (Gibco) supplemented with collagenase A $(1.5 \mathrm{mg} / \mathrm{ml}$, Roche, Machelen, Belgium) and DNAse I $(0.002 \mathrm{mg} / \mathrm{ml}$, SigmaAldrich, Diegem, Belgium), and $3 \mathrm{~min}$ in $0.05 \%$ trypsinethylenediaminetetraacetic acid (Invitrogen/Life Technologies Europe, Ghent, Belgium). The cell suspension obtained was then plated in six-well plates (Corning BV Life Sciences, Amsterdam, The Netherlands) at a cell density of $7-9 \times$ $10^{4}$ cells $/ \mathrm{cm}^{2}$ (one well for each fetal membrane) in RPMI medium supplemented with $10 \%$ fetal calf serum (FCS; Hyclone, GE Healthcare Europe GmbH, Diegem, Belgium), $100 \mathrm{U} / \mathrm{ml}$ penicillin (Invitrogen), and $100 \mathrm{mg} / \mathrm{ml}$ streptomycin (Invitrogen). For cell expansion and to establish a 
growth curve, cell culture medium was replaced every 3 days, and cells were split 1:2 and counted every 4 days. $\mathrm{mEFs}$ were cultured from $\mathrm{eGFP}^{+}$and $\mathrm{eGFP}^{-}$embryos following crossing of female wild-type C57BL/6 mice with male transgenic C57BL/6-eGFP mice, according to previously described procedures (21). For cell expansion, mEF medium [Dulbecco's modified Eagle's medium (DMEM) with $4.5 \mathrm{~g} / \mathrm{L}$ glucose and L-glutamine (Gibco) supplemented with $10 \% \mathrm{FCS}, 100 \mathrm{U} / \mathrm{ml}$ penicillin, and $100 \mathrm{mg} / \mathrm{ml}$ streptomycin] was replaced every 2-3 days, and cells were split 1:3 every 4-5 days. In order to establish a growth curve, $\mathrm{mEF}$ cultures were plated at $9 \times 10^{4}$ cells/ $\mathrm{cm}^{2}$, split 1:2, and counted every 4 days. BV-2 microglial cells (retrovirus-induced immortalized murine microglial cell line of unknown gender; kindly provided by Prof. R. Donato, University of Perugia, Italy) were cultured in DMEM supplemented with $10 \%$ FCS, $100 \mathrm{U} / \mathrm{ml}$ penicillin, and $100 \mathrm{mg} / \mathrm{ml}$ streptomycin. For cell culture, BV-2 medium was replaced every 2 days, and cells were split 1:5 every 3 days. All cell cultures were incubated in a humidified incubator at $37^{\circ} \mathrm{C}$ with $5 \% \mathrm{CO}_{2}$.

\section{Flow Cytometric Analysis}

Immunophenotyping of $\mathrm{eGFP}^{-}$mFMSC and $\mathrm{eGFP}^{-}$ $\mathrm{mEF}$ populations was performed using the following directly labeled antibodies: phycoerythrin (PE)-labeled rat anti-mouse stem cell antigen 1 (Sca1; FAB1226P; R\&D Systems, Abingdon, UK), PE-labeled mouse anti-mouse cluster of differentiation 90 (CD90; 12-0900-91; eBioscience, Vienna, Austria), PE-labeled rat anti-mouse CD44 (12-0441-81; eBioscience), PE-labeled rat anti-mouse major histocompatibility complex (MHC) class II (12-5321-82; eBioscience), PE-labeled rat anti-mouse CD184 (551966; BD, Erembodegem, Belgium), PE-labeled mouse anti-mouse MHC class $1(\mathrm{H} 2 \mathrm{~Kb}$; 553570; $\mathrm{BD})$, fluorescein isothiocyanate (FITC)-labeled rat anti-mouse CD45 (11-045182; eBioscience), FITC-labeled rat anti-mouse CD106 (11-1061-82; eBioscience), FITC-labeled rat anti-mouse CD31 (11-0311-82; eBioscience), FITC-labeled rat antimouse CD4 (11-0041-82; eBioscience), FITC-labeled rat anti-mouse CD8a (11-0081-82; eBioscience), and FITClabeled rabbit anti-mouse transforming growth factor- $\beta$ receptor II (TGF- $\beta$ RII, sc-1700; Santa Cruz Biotechnology, Dallas, TX, USA). Before staining, harvested cells were washed twice with PBS and suspended in PBS at a concentration of $2 \times 10^{6}$ cells $/ \mathrm{ml}$. For antibody staining, $1 \mu \mathrm{g}$ of antibody was added to $100 \mu \mathrm{l}$ of cell suspension for $30 \mathrm{~min}$ at $4^{\circ} \mathrm{C}$. Following incubation, cells were washed once with PBS, suspended in $1 \mathrm{ml}$ PBS, and analyzed by flow cytometry. For detection of eGFP transgene expression, harvested $\mathrm{eGFP}^{+} \mathrm{mFMSCs}$ and $\mathrm{eGFP}^{+} \mathrm{mEFs}$ were washed once with PBS, suspended in PBS, and directly analyzed by flow cytometry. For all analyses, cell viability was assessed by adding GelRed (1× final concentration; Biotum, Hayward, CA, USA) to the cell suspension immediately before flow cytometric analysis. All flow cytometric analyses were performed using an Epics XL-MCL analytical flow cytometer (Beckman Coulter, Suarlée, Belgium), and all data were analyzed using FlowJo flow cytometry data analysis software (FlowJo, Ashland, OR, USA).

\section{Cell Differentiation Assays}

For adipogenic differentiation, mFMSCs and mEFs were plated at $2 \times 10^{4}$ cells $/ \mathrm{cm}^{2}$ in 24-well plates (Corning) followed by culture in Mesenchymal Stem Cell Adipogenic Differentiation Medium (PT 3004; Lonza, Braine-l'Alleud, Belgium), according to the manufacturer's instructions. Control noninduced cells were kept in basal medium (RPMI 10\% FCS for mFMSCs and DMEM 10\% FCS for $\mathrm{mEFs})$. After 3 weeks of culture, cells were fixed with 4\% paraformaldehyde (Sigma) and stained with fresh Oil Red O solution (Sigma). For osteogenic differentiation, $\mathrm{mFMSCs}$ and $\mathrm{mEFs}$ were plated at $1 \times 10^{4} \mathrm{cells} / \mathrm{cm}^{2}$ in 24-well plates followed by culture in Mesenchymal Stem Cell Osteogenic Differentiation Medium (PT 3002; Lonza), according to the manufacturer's instructions. Control noninduced cells were kept in basal medium. After 3 weeks of culture, cells were fixed with $4 \%$ paraformaldehyde and stained with Alizarin Red S (Sigma). For chondrogenic differentiation, $2.5 \times 10^{5} \mathrm{mFMSCs}$ or $\mathrm{mEFs}$ were pelleted in $15-\mathrm{ml}$ conical tubes (Greiner Bio-One $\mathrm{GmbH}$, Frickenhausen, Germany) followed by culture in Mesenchymal Stem Cell Chondrocyte Differentiation Medium (PT 3003; Lonza) supplemented with $10 \mathrm{ng} / \mathrm{ml}$ TGF- $\beta 3$ (Sigma), according to the manufacturer's instructions. Control noninduced cells were kept in basal medium after pelleting. After 3 weeks of induction, cells were fixed with $4 \%$ paraformaldehyde and stained with Alcian blue (Sigma).

\section{mFMSC and mEF Coculture Experiments With BV-2 Cells}

mFMSCs and $\mathrm{mEFs}$ were plated at a concentration of $5 \times 10^{4}$ cells/well in a 24-well plate and allowed to adhere during overnight incubation. Next, in order to assess the effect of mFMSCs and mEFs on BV-2 microglial cells, $1 \times 10^{5} \mathrm{BV}-2$ cells were plated onto the confluent layer of mFMSCs or mEFs. Following $24 \mathrm{~h}$ of coculture, cultures were additionally stimulated with lipopolysaccharide (LPS, $1 \mu \mathrm{g} / \mathrm{ml}$; Invitrogen) + interferon- $\gamma($ IFN- $\gamma, 500 \mathrm{U} /$ $\mathrm{ml}$; R\&D Systems) to assess the effect of mFMSCs and mEFs on activated BV-2 microglial cells (28). After an additional $24 \mathrm{~h}$ of stimulation (or under control conditions), supernatant was harvested and analyzed for the presence of tumor necrosis factor- $\alpha$ (TNF- $\alpha$ ) and vascular endothelial growth factor (VEGF) by means of enzymelinked immunosorbent assay (ELISA), according to the 
manufacturer's instructions (murine TNF- $\alpha$ ELISA from Biolegend, London, UK; murine VEGF ELISA from Peprotech, London, UK).

\section{Cell Implantation Experiments}

Cell implantation in the CNS of immune-competent mice was performed under sterile conditions according to previously established procedures $(22,23)$. Briefly, eGFPexpressing $\mathrm{mFMSCs}$ and $\mathrm{mEFs}$ were harvested, washed with PBS, and resuspended at a concentration of approximately $41.6 \times 10^{6}$ cells $/ \mathrm{ml}$ in PBS. Directly prior to and posttransplantation, the cell number was recounted in order to control for the actual number of injected cells $\left(5 \times 10^{4}\right.$ cells in $1.2 \mu \mathrm{l}$ PBS $)$. For cell implantation, mice were anesthetized by an intraperitoneal injection of a ketamine $(80 \mathrm{mg} / \mathrm{kg}$; Pfizer, Puurs, Belgium $)+$ xylazine (16 mg/kg; Bayer Health Care, Diegem, Belgium) mixture in PBS and placed in a stereotactic frame (Stoelting, Dublin, Ireland). Next, a midline scalp incision was made, and a hole was drilled in the skull using a dental drill burr (Stoelting) at the following coordinates relative to bregma: $0.5 \mathrm{~mm}$ posterior and $2.2 \mathrm{~mm}$ lateral to the right side of the midline. Thereafter, an automatic microinjector pump (kdScientific, Holliston, MA, USA) with a 10- $\mu$ l Hamilton syringe was positioned above the exposed dura. A 30-gauge needle (Hamilton, Bonaduz, Switzerland), attached to the syringe, was stereotactically placed through the intact dura to a depth of $3.3 \mathrm{~mm}$. After $2 \mathrm{~min}$ of pressure equilibration, $5 \times 10^{4}$ cells of the respective populations were injected in a volume of $1.2 \mu \mathrm{l} \mathrm{PBS}$ at a speed of $0.7 \mu \mathrm{l} / \mathrm{min}$. The needle was retracted after another $4 \mathrm{~min}$ to allow pressure equilibration and to prevent backflow of the injected cell suspension. Next, the skin was sutured (Vicryl; Ethicon, Norderstedt, Germany), and a $0.9 \% \mathrm{NaCl}$ solution (Baxter, Lessen, Belgium) was administered subcutaneously in order to prevent dehydration while mice were placed under a heating lamp to recover.

\section{Histological Analysis: Immunofluorescence Staining}

Preparation of brain tissue for histological examination was performed according to previously optimized procedures $(22,23)$. Serial $10-\mu \mathrm{m}$-thick cryosections were obtained from the entire implant region using a Microm HM5000 cryostat (Microm GmbH, Walldorf, Germany), consecutively marked, and missing slides were noted. For further immunofluorescence analysis of tissue sections, antibody staining was performed as previously described $(22,23)$ using the following antibodies: (i) a rabbit antimouse Iba1 antibody (1:400 dilution, 019-19741; Wako, Neuss, Germany) in combination with an Alexa Fluor ${ }^{\circledR} 555$ labeled donkey anti-rabbit secondary antibody (1:1,000 dilution; A31572; Invitrogen), (ii) a mouse anti-mouse GFAP antibody (1:400 dilution, mab377; Merck Millipore, Merck KGaA, Darmstadt, Germany) in combination with an
Alexa Fluor ${ }^{\circledR}$ 350-labeled goat anti-mouse secondary antibody (1:200 dilution, A11068; Invitrogen), (iii) a rabbit antimouse S100b antibody (1:400 dilution, ab52642; Abcam Cambridge, UK) in combination with an Alexa Fluor ${ }^{\circledR} 555$ labeled donkey anti-rabbit secondary antibody (1:1,000 dilution, A-31572; Invitrogen), (iv) a rabbi anti-mouse CD31 antibody (1:50, AB28364; Abcam) in combination with a Texas Red-labeled goat anti-rabbit secondary antibody (1:1,000, AB6719; Abcam). Antibody stainings were performed using the following single or double staining combinations: (i) Ibal ( $n=2$ per cell grafted mouse brain), (ii) S100b + GFAP ( $n=2$ per cell grafted mouse brain), (iii) CD31 ( $n=1$ per cell grafted mouse brain). In brief, slides were rinsed for $5 \mathrm{~min}$ in Tris-buffered saline (TBS; Merck, Overijse, Belgium) and subsequently incubated for $30 \mathrm{~min}$ in $0.1 \%$ Triton-X (Merck) in TBS. Next, slides were washed, followed by a 1-h incubation at room temperature in TBS + blocking serum [for Iba-1 staining, TBS $+20 \%$ donkey serum from Jackson ImmunoResearch (01-000-121; Newmarket, Suffolk, UK) for S100b/GFAP staining, TBS $+20 \%$ donkey serum, $20 \%$ goat serum from Jackson ImmunoResearch (005-000-121) and 1\% unconjugated goat anti-mouse Ig from Jackson ImmunoResearch (115-007-003); for CD31 staining, TBS $+20 \%$ goat serum]. Next, blocking solution was removed, and slides were incubated overnight at $4{ }^{\circ} \mathrm{C}$ with the primary antibodies diluted in TBS $+10 \%$ (w/v) milk powder (Fluka BioChemika, Sigma-Aldrich, Diegem, Belgium). Next, slides were washed with TBS $(4 \times 3 \mathrm{~min})$ and incubated for $1 \mathrm{~h}$ at room temperature on a horizontal shaker (at 60 rpm; Bibby Scientific Limited, Stone, Staffordshire, UK) with the secondary antibodies diluted in TBS + 10\% (w/v) milk powder. Thereafter, slides were washed with TBS $(4 \times 3 \mathrm{~min})$, and a 20 -min nuclear staining was performed using 4',6-diamidino-2-phenylindole (DAPI, 1:1000 dilution; Sigma-Aldrich). Following final washing with TBS $(3 \times 2 \mathrm{~min})$, stained slides were mounted using Prolong Gold Antifade Reagent (P36930; Invitrogen), and images were acquired using an Olympus BX51 microscope (Olympus, Aartselaar, Belgium) equipped with an Olympus DP71 digital camera. Olympus CellSense software was used for image acquisition.

\section{Histological Analysis: Quantitative Analysis}

Quantitative analysis of cell graft survival, glial cell responses, and angiogenesis was performed using NIH ImageJ analysis software (Bethesda, MD, USA) and TissueQuest immunofluorescence analysis software (TissueGnostics $\mathrm{GmbH}$, Vienna, Austria) as previously described $(22,23)$, allowing determination of the following parameters: (i) the $\mathrm{mFMSC} / \mathrm{mEF}$ graft site volume in $\mathrm{mm}^{3}$ (one data count per cell graft analyzed), (ii) the density (in cells $/ \mathrm{mm}^{3}$ ) and absolute number of eGFP $+\mathrm{mFMSCs} / \mathrm{mEFs}$ within the graft site (four estimates per cell graft analyzed), 
(iii) the $\mathrm{mFMSC} / \mathrm{mEF}$ cell graft survival provided in absolute numbers and in percent calculated to the initial number of grafted $\mathrm{eGFP}^{+}$viable $\mathrm{mFMSCs} / \mathrm{mEFs}$ (four estimates per cell graft analyzed), (iv) the density (in cells $/ \mathrm{mm}^{3}$ ) and absolute number of $\mathrm{Iba1}^{+}$microglia within the $\mathrm{mFMSC} /$ $\mathrm{mEF}$ graft site (four estimates per cell graft analyzed), (v) the density (in cells $/ \mathrm{mm}^{3}$ ) of $\mathrm{Iba}^{+}$microglia within the $\mathrm{mFMSC} / \mathrm{mEF}$ graft site border as determined by a region extending $100 \mu \mathrm{m}$ from the $\mathrm{mFMSC} / \mathrm{mEF}$ graft site (two estimates per cell graft analyzed), (vi) the density (in cells $/ \mathrm{mm}^{3}$ ) of $\mathrm{S} 100 \mathrm{~b}^{+}$astrocytes within the $\mathrm{mFMSC} / \mathrm{mEF}$ graft site border (two estimates per cell graft analyzed), (vii) the degree of $\mathrm{GFAP}^{+}$astrogliosis within the mFMSC/ $\mathrm{mEF}$ graft site border provided as percent astrogliosis (two estimates per cell graft analyzed), and (viii) the density (in cells $/ \mathrm{mm}^{2}$ ) of $\mathrm{CD} 31^{+}$blood vessels within the mFMSC/ $\mathrm{mEF}$ graft (one data count per cell graft analyzed).

\section{Statistical Analyses}

All statistical analyses were performed using the IBM SPSS version 20 statistical package (Armonk, NY, USA). To examine differences in in vitro cell growth (day 0, day 4 , and day 8 postplating) between mFMSC and $\mathrm{mEF}$ cultures, a mixed model with repeated measures and a first-order autoregressive covariance structure was fitted. To evaluate differences in in vitro TNF- $\alpha /$ VEGF secretion, both outcomes were first transformed using an inverselogistic equation. A one-way ANOVA was then performed on these transformed variables, and several post hoc tests were used to assess statistically significant differences (highest $p$ value is reported). Post hoc tests were for VEGF the Tukey HSD, Scheffe, Bonferroni, and Tamhane tests and for TNF- $\alpha$ the Tamhane, Dunnet T3, and GamesHowell tests. To evaluate differences in graft site volume, total cell number within the graft site, number of $\mathrm{eGFP}^{+}$cells within the graft site, percent cell graft survival, ratio of microglia versus $\mathrm{eGFP}^{+}$cells within the graft site, density of microglia in the graft site surrounding, density of astrocytes in the graft site surrounding, percent of astrogliosis and density of blood vessels within the graft site, generalized estimating equations (30) taking into account repeated measures were used. This method does not require a priori specification of the association structure. The obtained $p$ values were corrected using the false-discovery rate control (3). For all comparisons, a value of $p<0.05$ was considered to be statistically significant.

\section{RESULTS}

\section{Phenotypical Characterization of mFMSC and $\mathrm{mEF}$ Cultures}

mFMSCs and mEFs were isolated from wild-type C57BL/6 mice or from transgenic C57BL/6-eGFP mice, as described in the Materials and Methods section. As shown in Figure 1A, a and b (main images), both mFMSCs and $\mathrm{mEFs}$ are adherently cultured cell populations displaying a typical fibroblast-like morphology. When mFMSCeGFP and mEF-eGFP were isolated from C57BL/6-eGFP transgenic mice, both cell populations uniformly displayed high eGFP expression as measured by flow cytometry (Fig. 1A, a and b, inset images). Further flow cytometric immunophenotyping of mFMSCs and mEFs characterized both cell populations as follows: $\mathrm{Sca}^{+}, \mathrm{CD}_{4}{ }^{+}, \mathrm{CD} 90^{+(\mathrm{low})}$, $\mathrm{CD}^{-}{ }^{-}, \mathrm{CD}_{106}^{-}, \mathrm{CD}^{-} 5^{-}, \mathrm{CD}_{184}{ }^{-}, \mathrm{CD}^{-}, \mathrm{CD}^{-}, \mathrm{TGF} \beta$ $\mathrm{RII}^{-}, \mathrm{MHCI}^{-}$, and $\mathrm{MHCII}^{+(\text {low })}$ (Fig. 1A, c and d). Of note, these embryonic stromal cell populations are phenotypically slightly different from adult C57BL/6 murine bone marrowderived mesenchymal stromal cells (mBMMSCs), as the latter were previously characterized as $\mathrm{Sca}^{+ \text {(high) }}{ }^{\mathrm{CD}} 44^{+(\text {(high)}}$, $\mathrm{CD}^{-}{ }^{-}, \mathrm{CD}^{-} 1^{-}, \mathrm{CD}_{106}^{-}, \mathrm{CD}^{-} 5^{-}, \mathrm{CD}_{184}^{-}, \mathrm{CD}^{-}, \mathrm{CD}^{-}$,

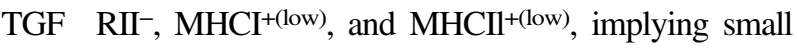
phenotypic variations between fibroblast-like cell types derived from adult or embryonic tissues (data not shown).

\section{Functional Characterization of $m F M S C$ and $m E F$}

First, we evaluated the proliferative capacity of the isolated $\mathrm{mFMSC}$ and $\mathrm{mEF}$ populations (Fig. 1B). While $\mathrm{mEF}$ cultures can easily be expanded in vitro $(p<0.001)$, mFMSCs did not display any proliferation. Moreover, in the current setup, we were unable to maintain mFMSCs in culture for more than 2-3 weeks due to cell detachment followed by cell death $(p<0.001)$. Next, we evaluated the in vitro differentiation potential of mFMSCs and mEFs. For this, both cell types were cultured in commercially available adipogenic, osteogenic, and chondrogenic differentiation media. As shown in Figure 1C (left panels), mFMSCs displayed a reduced differentiation potential, limited to a low osteogenic commitment, compared to $\mathrm{mEFs}$, which were able to differentiate to both adipogenic and osteogenic lineages. No spontaneous adipogenic or osteogenic differentiation was observed for nonstimulated mFMSC and mEF cultures (Fig. 1C, right panels). Neither mFMSCs nor mEFs underwent chondrogenic differentiation upon culture in chondrogenic differentiation medium (data not shown).

\section{Immunomodulating Effect of mFMSCs and $m E F$ s on $B V-2$ Microglia}

In this part of the study, we aimed to investigate whether mFMSCs or mEFs could exert an immunomodulating effect on the activation of the C57BL/6-derived BV-2 microglial cell line. To this end, we followed the experimental setup as described in Figure 1D, a (upper scheme). As shown in Figure 1D, b (lower graph), BV-2 cells readily produce high amounts of TNF- $\alpha$ upon stimulation with LPS/IFN- $\gamma(p<0.001$ for BV-2 vs. BV-2+LPS/IFN- $\gamma)$. mFMSCs do not produce significant amounts of TNF- $\alpha$, nor upon stimulation with LPS/IFN- $\gamma$ or coculture with $\mathrm{BV}-2$ cells. However, when mFMSC/BV-2 cocultures are 


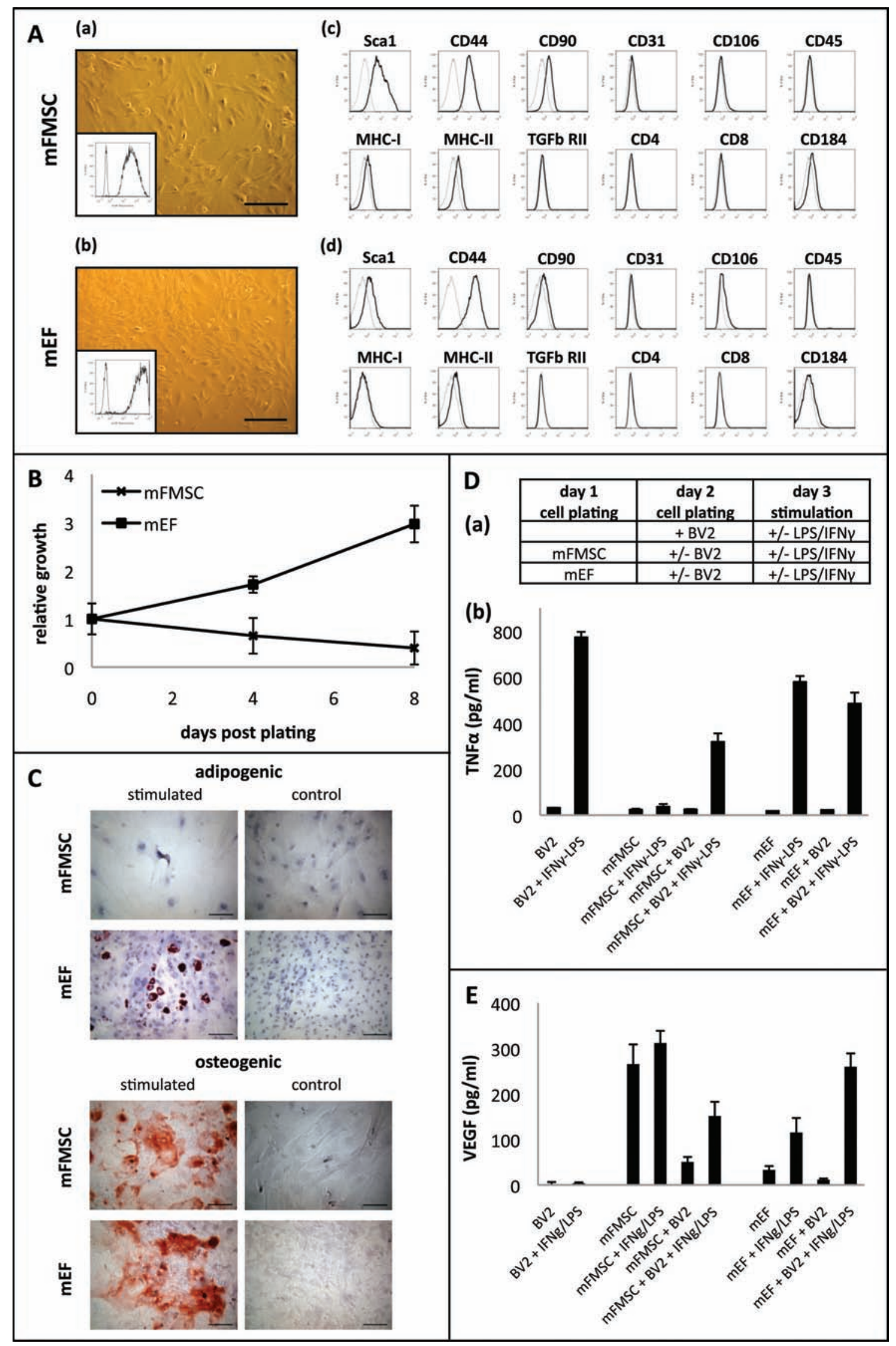


stimulated with LPS/IFN- $\gamma$, a reduced production of TNF- $\alpha$ by BV-2 cells can be noted $(p<0.001$ for mFMSC + BV-2 + LPS/IFN- $\gamma$ vs. BV-2 + LPS/IFN- $\gamma$ ). While mEFs alone or upon coculture with BV-2 cells do not produce significant amounts of TNF- $\alpha$, stimulation of mEFs with LPS/IFN- $\gamma$ leads to a significant increase in TNF- $\alpha$ production $(p<0.001$ for $\mathrm{mEFs}$ vs. $\mathrm{mEFs}+\mathrm{LPS} / \mathrm{IFN}-\gamma)$. This observation indicates that, independent of their embryonic fibroblast-like origin, both mFMSCs and mEFs behave differently upon encountering immune stimuli. Nevertheless, even though mEFs can produce significant amounts of TNF- $\alpha$, in the event of LPS/IFN- $\gamma$ stimulation of $\mathrm{mEF} /$ BV-2 cocultures, an overall reduced production of TNF- $\alpha$ by BV-2 (or $\mathrm{mEF}$ ) cells can be noted $(p=0.001$ for $\mathrm{mEF}+\mathrm{BV}-2+\mathrm{LPS} / \mathrm{IFN}-\gamma$ vs. BV- $2+\mathrm{LPS} / \mathrm{IFN}-\gamma)$. Based on the presented data, we conclude that both mFMSCs and $\mathrm{mEFs}$ are able to partially modulate in vitro activation of the BV-2 microglial cell line.

\section{Differential Production of VEGF by mFMSCs and $m E F s$ Under Normal and Inflammatory Conditions}

In this part of the study, we investigated the in vitro secretion of VEGF by mFMSC and mEF cultures in the absence or presence of (activated) BV-2 microglial cells (see Fig. 1D, a, upper scheme, for experimental setup). As shown in Figure 1E, mFMSC cultures secrete significantly higher levels of VEGF in comparison to $\mathrm{mEF}$ cultures $(p<0.001$ for mFMSCs vs. mEFs). Unexpectedly, in the presence of (activated) BV-2 microglia,
mFMSCs reduce their production of VEGF $(p<0.001$ for mFMSCs vs. mFMSCs $+\mathrm{BV}-2$ and $p=0.004$ for mFMSCs vs. mFMSCs + BV-2 + LPS/IFN- $\gamma$ ). In contrast, mEFs display an increased VEGF production upon application of proinflammatory stimuli $(p<0.001$ for $\mathrm{mEFs}$ vs. $\mathrm{mEFs}+\mathrm{LPS} / \mathrm{IFN}-\gamma)$, which is further increased by the presence of activated BV-2 microglia $(p<0.001$ for $\mathrm{mEFs}+\mathrm{LPS} / \mathrm{IFN}-\gamma$ vs. $\mathrm{mEFs}+\mathrm{BV}-2+\mathrm{LPS} / \mathrm{IFN}-\gamma)$. Based on the presented data, we conclude that mFMSCs and $\mathrm{mEFs}$ might display different angiogeneic properties upon in vivo grafting into an inflammatory environment.

\section{Quantitative Analysis of mFMSC and mEF Graft Survival Following Intracerebral Transplantation}

Next, we aimed to investigate the in vivo behavior of mFMSCs and mEFs upon autologous grafting in the CNS of immune-competent mice. To this end, $5 \times 10^{4}$ mFMSC-eGFP or $5 \times 10^{4} \mathrm{mEF}-\mathrm{eGFP}$ were grafted into the CNS of syngeneic C57BL/6 mice ( $n=5$ for each group). Histological analyses of brain tissue from cellgrafted mice were performed at day 14 postimplantation. For this, cryosections of the whole graft site area were prepared and screened for the presence of eGFP-expressing mFMSC or mEF implants. From the representative images provided in Figure 2A, it is clear that eGFP-expressing cellular grafts could be detected 2 weeks after implantation (cell grafts were identified in 4/5 mice transplanted with mFMSC-eGFP and in 5/5 mice transplanted with mEFeGFP). Notably, the graft site volume (i.e., the volumetric

\section{FACING PAGE}

Figure 1. In vitro characterization of mFMSCs and mEFs. (A) Bright field images of in vitro cultured mFMSCs (a) and mEFs (b). Scale bar: $200 \mu \mathrm{m}$. Inset images: flow cytometric analysis of eGFP expression. Thin line histogram: FL-1 fluorescence of control mFMSCs (a) and mEFs (b). Bold line histogram: eGFP fluorescence of mFMSC-eGFP (a) and mEF-eGFP (b). Immunophenotypical analysis of mFMSCs (c) and mEFs (d). Thin line histogram: unstained control. Bold line histogram: specific antibody staining. Representative images were chosen from two independent immune phenotyping experiments for mFMSCs and mEFs. Sca1, stem cell antigen; CD44, cluster of differentiation 44; MHC, major histocompatibility complex; TGF- $\beta$ RII, transforming growth factor- $\beta$ receptor II. (B) In vitro growth of mFMSC(-eGFP) $(n=9)$ and $\mathrm{mEF}(-\mathrm{eGFP})(n=3)$ cultures. The counted cell number at plating was set to 1 for data analysis. The values are given as the average \pm standard deviation. Results indicate an increase in cell number over time for $\mathrm{mEF}$ cultures $(p<0.001)$ and a decrease in cell number over time for mFMSC cultures $(p<0.001)$. (C) In vitro differentiation potential of mFMSCs and mEFs. Adipogenic differentiation assay: lipid droplets marked by Oil Red $\mathrm{O}$ staining for neutral lipids. Osteogenic differentiation assay: calcium deposition evidenced by Alizarin Red staining (scale bar: $100 \mu \mathrm{m}$ ). Representative images were chosen from two independent differentiation experiments for mFMSCs and mEFs. (D) In vitro evaluation of immunomodulation properties of mFMSCs and mEFs. (a) Schematic overview of the experimental outline. (b) Bar chart shows TNF- $\alpha$ secretion by BV-2 cells alone, after stimulation with LPS/IFN- $\gamma$ and after coculture with mFMSCs or mEFs. TNF- $\alpha$ production has been quantified by ELISA on cell culture supernatants $24 \mathrm{~h}$ after LPS/IFN- $\gamma$ stimulation. Presented results are given as average \pm standard deviation for two independent stimulation experiments for mFMSCs and $\mathrm{mEFs}$, with each experiment performed in quadruplicate (for each condition, $n=8$ ). Results indicate (i) that BV-2 cells produce significant levels of TNF- $\alpha$ upon stimulation with LPS/IFN- $\gamma(p<0.001)$, (ii) that mFMSCs reduce the level of TNF- $\alpha$ production by LPS/IFN- $\gamma$-stimulated BV-2 cells $(p<0.001)$, (iii) that mEFs produce significant levels of TNF- $\alpha$ upon stimulation with LPS/IFN- $\gamma(p<0.001)$, and (iv) that despite intrinsic potential to produce TNF- $\alpha$, mEFs still reduce the level of TNF- $\alpha$ production by LPS/IFN- $\gamma$-stimulated BV-2 cells $(p=0.001)$. (E) In vitro VEGF production by mFMSCs and mEFs. Bar chart shows VEGF production by mFMSCs or mEFs alone, after stimulation with LPS/IFN- $\gamma$ in the presence or absence of BV-2 cells. VEGF production has been quantified by ELISA on cell culture supernatants $24 \mathrm{~h}$ after LPS/IFN- $\gamma$ stimulation. Presented results are given as average \pm standard deviation for two independent stimulation experiments for mFMSCs and mEFs, with each experiment performed in quadruplicate (for each condition, $n=8$ ). Results indicate (i) that mFMSCs produce significant levels of VEGF $(p<0.001)$, (ii) that both nonactivated and LPS/IFN- $\gamma$-stimulated BV-2 cells can suppress VEGF production by mFMSCs $(p<0.001$ and $p=0.004)$, and (iii) that mEFs display an increased level of VEGF production upon stimulation with LPS/IFN- $\gamma(p<0.001)$, which is further increased in the presence of LPS/IFN- $\gamma$-stimulated BV-2 cells $(p<0.001)$. 


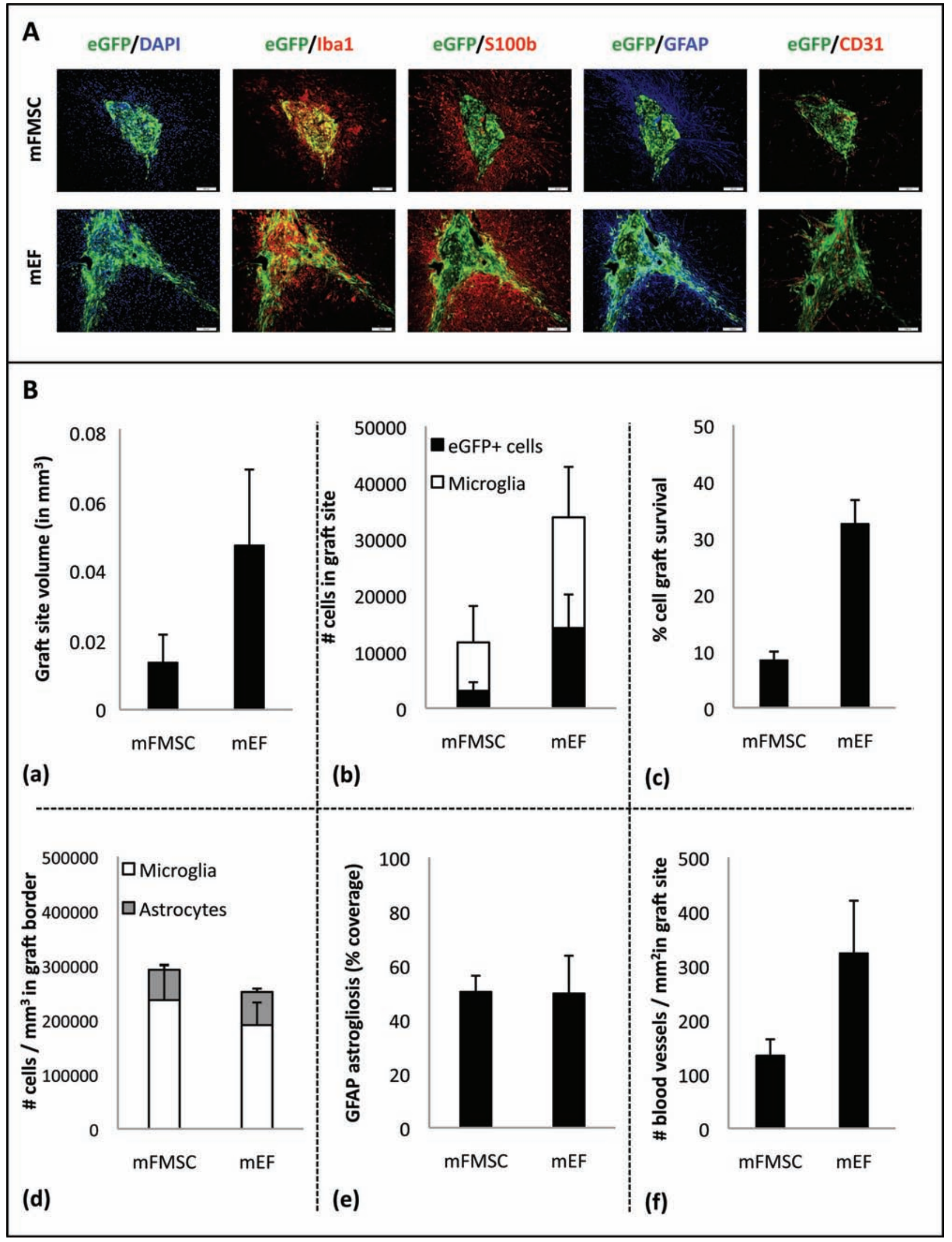


area containing eGFP-expressing cells) (Fig. 2B, a) and the total number of cells within the graft site (comprising both eGFP-expressing grafted cells and microglia) (Fig. 2B, b) are significantly smaller for mFMSC grafts compared to mEF grafts (respectively, $p<0.001$ and $p=0.002$ ). Subsequently, it was noted that the number of $\mathrm{eGFP}^{+}$cells within mFMSC grafts was significantly lower than the number of $\mathrm{eGFP}^{+}$cells within mEF grafts $(p<0.001)$ (Fig. 2B, b). Comparing with the initial number of grafted cells $\left(5 \times 10^{4}\right.$ cells $)$, a significantly different degree of cell graft survival was calculated for mFMSC grafts $(8 \% \pm$ $4 \%)$ as opposed to $\mathrm{mEF}$ grafts $(32 \% \pm 14 \% ; p<0.001)$ (Fig. 2B, c).

\section{Quantitative Analysis of Glial Cell Responses Following mFMSC and mEF Grafting}

In our preceding studies regarding $\mathrm{mMSC}$ and $\mathrm{mEF}$ grafting in the CNS of immune-competent mice, we noted that mMSC and mEF grafts became highly infiltrated by microglia (at week 2 postgrafting, up to $50-80 \%$ of cells within the graft site are microglia) and surrounded by microglia and astrocytes $(4,5,10,21)$. From the representative Iba1/eGFP, S100b/eGFP, and GFAP/eGFP images provided in Figure 2A, it is clear that mFMSC grafts, too, become highly infiltrated by microglia and surrounded by both microglia and astrocytes. As shown in Figure 2B, b microglia comprise, respectively, $75 \% \pm 6 \%$ and $58 \% \pm$ $3 \%$ of the total number of cells within a mFMSC and $\mathrm{mEF}$ graft site, with the ratio of microglia to $\mathrm{eGFP}^{+}$cells being significantly higher for mFMSC grafts than for $\mathrm{mEF}$ grafts (respectively, $2.9 \pm 0.8$ and $1.5 \pm 0.3, p=0.001$ ). The latter can, however, presumably be explained by the lower degree of cell survival observed for mFMSC grafts compared to $\mathrm{mEF}$ grafts. For the area surrounding the graft site, no difference in the density of $\mathrm{Iba}^{+}$microglia $(p=0.205)$ and $\mathrm{S}_{100 b^{+}}$astrocytes $(p=0.358)$ was observed between mFMSC and mEF grafts (Fig. 2B, d). Additionally, no difference in the degree of $\mathrm{GFAP}^{+}$astrogliosis was observed around mFMSC and mEF grafts $(p=0.924)$ (Fig. 2B, e). Therefore, despite the lower degree of mFMSC graft survival compared to $\mathrm{mEF}$ grafts, no differences were observed in both degree and organization of endogenous glial cell responses.

\section{Quantitative Analysis of Neoangiogenesis Within mFMSC and mEF Grafts}

Long-term survival of cellular grafts in vivo relies on the ability of the grafted cell type to support neoangiogenesis. Although the precise mode of action has not yet been fully unraveled, it is well recognized that fibroblasts play a major role in physiological and pathological angiogenesis. Therefore, we additionally determined neoangiogenesis in mFMSC and mEF grafts. As shown by the representative CD31/eGFP images in Figure 2A and their graphical representation in Figure 2B, f, a significantly lower blood vessel density was observed within mFMSC compared to $\mathrm{mEF}$ cell grafts $(p<0.001)$. This observation is fully in line with our in vitro results regarding the respectively reduced/increased VEGF production by mFMSCs and mEFs under inflammatory conditions (Fig. 1E).

\section{DISCUSSION}

Fibroblast cells of mesenchymal origin are considered as highly attractive candidates for cell-grafting experiments in the CNS. Although the main aim in this strategy is not direct cell replacement therapy, fibroblast(-like) cells might exert beneficial effects by means of bystander therapy. For example, exploitation of their immunomodulating, regeneration-inducing, and/or antiapoptotic capacity has been well described in multiple preclinical disease models of CNS and peripheral injury $(1,5,9,25,27)$. Additionally, mesenchymal cells are attractive candidates as carriers for therapeutic proteins in vivo, a strategy that is extensively investigated at the moment $(24,26)$. Nevertheless, little is currently known about the actual in vivo fate of the grafted cells. In our preceding studies, we already described that both mFMSCs and mEFs become surrounded and infiltrated by microglia and surrounded by astrocytes. In this study, we observed a similar immune recognition pattern rin vivo using mFMSCs as an extraembryonic mesenchymal

\section{FACING PAGE}

Figure 2. Histological analysis of mFMSC-eGFP and mEF-eGFP grafts in the CNS of immune-competent mice. (A) Representative immunofluorescent images of the histological analysis of mFMSC-eGFP and mEF-eGFP grafts. Direct eGFP fluorescence (in green) combined with (from left to right) nuclear staining with DAPI (in blue), immunofluorescent staining for microglia (Iba1, in red), astrocytes (S100b, in red), astrogliosis (GFAP, in blue), and blood vessels (CD31, in red) at week 2 postimplantation. Representative images were chosen from multiple stained slides $(n=2$ for eGFP/Iba1 and eGFP/S100b/GFAP combinations, and $n=1$ for eGFP/CD31 combination) per cell graft analyzed ( $n=4$ for mFMSC-eGFP grafts and $n=5$ for mEF grafts). Scale bars: $100 \mu \mathrm{m}$. (B) Quantitative analysis of histological images. For each graph, data are presented as mean \pm standard deviation for multiple cell grafts analyzed ( $n=4$ for mFMSC-eGFP grafts and $n=5$ for mEF grafts). (a) Graft site volume in $\mathrm{mm}^{3}$. Significant difference between mFMSCs and mEFs $(p<0.001)$. (b) Absolute number of Iba1 ${ }^{+}$microglia (white bars) and eGFP ${ }^{+}$cells (black bars) within the graft site. Significant difference between mFMSCs and mEFs with regard to total cell density $(p=0.002)$ and significant difference between mFMSCs and mEFs with regard to total number of surviving $\mathrm{eGFP}^{+}$cells. (c) Cell graft survival given in percent. Significant difference between mFMSCs and mEFs $(p<0.001)$. (d) Density of $\mathrm{Iba}^{+}$microglia (white bars) and S100b ${ }^{+}$astrocytes (gray bars) within the implant border. (e) Degree of astrogliosis within the implant border (presented as percent coverage based on GFAP staining). (f) Density of CD31+ blood vessels within the implant site. Significant difference between mFMSCs and mEFs $(p<0.001)$. 
cell population. However, our data do highlight several differences between mEFs and mFMSCs. First, in contrast to mEFs, mFMSCs do not display proliferation in vitro. Currently, we do not have a valid explanation for this observation, although it remains to be elucidated whether the used culture medium for mFMSCs can be further optimized. With regard to this matter, it should be noted here that murine fetal membranes are developmentally organized differently compared to human fetal membranes (11) and therefore might benefit from the use of a different expansion medium (in this study, we used expansion medium that has proven effective for hFMSCs). Second, for our cultured mFMSCs, we were unable to demonstrate in vitro trilineage differentiation, a feature that is also under debate for hFMSCs (19). However, as $\mathrm{mEFs}$ displayed differentiation into adipogenic and osteogenic lineage, we believe our in vitro differentiation protocol to be satisfactory. Therefore, murine development may have imposed distinct features to mFMSCs and $\mathrm{mEFs} / \mathrm{mMSCs}$, despite all being of mesenchymal origin. Further research will have to address this topic. Of note, a recently published study also attributed distinct immunomodulatory and migratory mechanisms to human mesenchymal cells derived from different tissues $(15,20)$. Third, while $\mathrm{mEF}$ cultures are responsive to LPS/IFN- $\gamma$ signaling, mFMSC cultures are unresponsive, at least with regard to TNF- $\alpha$ production. Although further investigation will be needed to determine the presence and/or absence of toll-like receptors on both cell populations and their responsiveness to stimuli of the immune system, the unresponsiveness of mFMSCs can potentially be viewed in light of protecting the embryo against inflammatory signaling during maternal inflammation. However, with regard to the intrinsic capacity of mFMSCs and mEFs to suppress TNF- $\alpha$ by activated microglia, we did not observe significant differences between both cell populations. This observation can, however, be explained by the fact that mostif not all-cells of mesenchymal origin display this feature in vitro (13). Fourth, despite the absence of a manifest difference in the in vivo immune response triggered against mFMSC or mEF grafts, it was clearly noted that mFMSCs display a lower degree of cell graft survival compared to $\mathrm{mEF}$ grafts. Although further research will be needed, our study indicated differences in the capacity of mFMSCs and mEFs to produce VEGF. The ability to support neoangiogenesis is a major prerequisite for cell graft survival as-in most cases-cell transplantation is performed without any structural support. Unexpectedly, we first noted that mFMSCs produce high levels of VEGF, while mEFs do not. However, upon coculture of mFMSCs with (activated) microglial cells, a reduction in VEGF secretion was noted. In contrast, mEFs are triggered to produce high levels of VEGF upon coculture with activated microglial cells. As we have shown in a preceding study that microglia within MSC grafts display an M1oriented phenotype (10), we here suggest that the activation status of graft-infiltrating microglia might trigger different angiogenic responses in different fibroblast-like cell types, thereby potentially correlating the lower degree of mFMSC graft survival compared to mEF graft survival. Our work thus implies that further research using advanced cellular and molecular analysis tools will have to reveal the complex signaling between mesenchymal cell grafts, microglia, astrocytes, and especially endothelial (progenitor) cells in vivo. The latter has also been suggested by in vitro observations describing a cross talk between mesenchymal stromal cells and endothelial progenitor cells under certain conditions $(6,18)$.

Concluding this study, we have compared the behavior of mFMSCs and mEFs in vitro and in vivo following grafting in the CNS. The presented data indicate significant differences in the ability of both cell populations to respond to inflammatory stimuli, resulting in differential production of TNF- $\alpha$ and VEGF under normal and inflammatory conditions. Although further investigation is needed, these apparent differences may lie at the basis of the presence or absence of functional recovery following grafting of different fibroblast cell types in animal models of CNS (and other) injuries. Our data thus underscore the need for a thorough characterization of the cell populations used in cell transplantation studies, as well as the need for comparative cell transplantation studies with similar, but developmentally different, cell populations. Only the latter will lead to a better understanding of the beneficial effects observed in many preclinical studies that currently face difficulties when being translated to human clinical applications.

ACKNOWLEDGMENTS: This work was supported by research grants G.0136.11 and G.0130.11 (granted to Z.B. and P.P.) of the Fund for Scientific Research-Flanders (FWO-Vlaanderen, Belgium) and in part by a Methusalem research grant from the Flemish government (granted to Z.B. and H.G.). Nathalie De Vocht and Chloé Hoornaert hold a Ph.D.-studentship from the FWO-Vlaanderen. Debbie Le Blon holds a Ph.D.-studentship from the Flemish Institute for Science and Technology (IWT). The authors declare no conflicts of interest.

\section{REFERENCES}

1. Atoui, R.; Chiu, R. C. Concise review: Immunomodulatory properties of mesenchymal stem cells in cellular transplantation: Update, controversies, and unknowns. Stem Cells Transl. Med. 1(3):200-205; 2012.

2. Barry, F. P.; Murphy, J. M. Mesenchymal stem cells: Clinical applications and biological characterization. Int. J. Biochem. Cell Biol. 36(4):568-584; 2004.

3. Benjamini, Y.; Hochberg, Y. Controlling the false discovery rate - A practical and powerful approach to multiple testing. J. R. Statist. Soc. B. 57(1):289-300; 1995.

4. Bergwerf, I.; De Vocht, N.; Tambuyzer, B.; Verschueren, J.; Reekmans, K.; Daans, J.; Ibrahimi, A.; Van Tendeloo, V.; Chatterjee, S.; Goossens, H.; Jorens, P. G.; Baekelandt, V.; Ysebaert, D.; Van Marck, E.; Berneman, Z. N.; Van der 
Linden, A.; Ponsaerts, P. Reporter gene-expressing bone marrow-derived stromal cells are immune-tolerated following implantation in the central nervous system of syngeneic immunocompetent mice. BMC Biotechnol. 9:1; 2009.

5. Bergwerf, I.; Tambuyzer, B.; De Vocht, N.; Reekmans, K.; Praet, J.; Daans, J.; Chatterjee, S.; Pauwels, P.; Van der Linden, A.; Berneman, Z. N.; Ponsaerts, P. Recognition of cellular implants by the brain's innate immune system. Immunol. Cell Biol. 89(4):511-516; 2011.

6. Bidarra, S. J.; Barrias, C. C.; Barbosa, M. A.; Soares, R.; Amedee, J.; Granja, P. L. Phenotypic and proliferative modulation of human mesenchymal stem cells via crosstalk with endothelial cells. Stem Cell Res. 7(3):186-197; 2011.

7. Bieback, K.; Wuchter, P.; Besser, D.; Franke, W.; Becker, M.; Ott, M.; Pacher, M.; Ma, N.; Stamm, C.; Kluter, H.; Muller, A.; Ho, A. D. Mesenchymal stromal cells (MSCs): Science and f(r)iction. J. Mol. Med. 90(7):773-782; 2012.

8. Cargnoni, A.; Gibelli, L.; Tosini, A.; Signoroni, P. B.; Nassuato, C.; Arienti, D.; Lombardi, G.; Albertini, A.; Wengler, G. S.; Parolini, O. Transplantation of allogeneic and xenogeneic placenta-derived cells reduces bleomycininduced lung fibrosis. Cell Transplant. 18(4):405-422; 2009.

9. Dailey, T.; Tajiri, N.; Kaneko, Y.; Borlongan, C. V. Regeneration of neuronal cells following cerebral injury. Front. Neurol. Neurosci. 32:54-61; 2013.

10. De Vocht, N.; Lin, D.; Praet, J.; Hoornaert, C.; Reekmans, K.; Le Blon, D.; Daans, J.; Pauwels, P.; Goossens, H.; Hens, N.; Berneman, Z.; Van der Linden, A.; Ponsaerts, P. Quantitative and phenotypic analysis of mesenchymal stromal cell graft survival and recognition by microglia and astrocytes in mouse brain. Immunobiology 218(5):696-705; 2013.

11. Dobreva, M. P.; Pereira, P. N.; Deprest, J.; Zwijsen, A. On the origin of amniotic stem cells: Of mice and men. Int. J. Dev. Biol. 54(5):761-777; 2010.

12. Evangelista, M.; Soncini, M.; Parolini, O. Placenta-derived stem cells: New hope for cell therapy? Cytotechnology 58(1): 33-42; 2008.

13. Haniffa, M. A.; Collin, M. P.; Buckley, C. D.; Dazzi, F. Mesenchymal stem cells: The fibroblasts' new clothes? Haematologica 94(2):258-263; 2009.

14. Ilancheran, S.; Moodley, Y.; Manuelpillai, U. Human fetal membranes: A source of stem cells for tissue regeneration and repair? Placenta 30(1):2-10; 2009.

15. Liu, K. J.; Wang, C. J.; Chang, C. J.; Hu, H. I.; Hsu, P. J.; Wu, Y. C.; Bai, C. H.; Sytwu, H. K.; Yen, B. L. Surface expression of HLA-G is involved in mediating immunomodulatory effects of placenta-derived multipotent cells (PDMCs) towards natural killer lymphocytes. Cell Transplant. 20(11-12):1721-1730; 2011.

16. Manuelpillai, U.; Moodley, Y.; Borlongan, C. V.; Parolini, O. Amniotic membrane and amniotic cells: Potential therapeutic tools to combat tissue inflammation and fibrosis? Placenta 32(Suppl 4):S320-S325; 2011.

17. Manuelpillai, U.; Tchongue, J.; Lourensz, D.; Vaghjiani, V.; Samuel, C. S.; Liu, A.; Williams, E. D.; Sievert, W. Transplantation of human amnion epithelial cells reduces hepatic fibrosis in immunocompetent $\mathrm{CCl}(4)$-treated mice. Cell Transplant. 19(9):1157-1168; 2010.

18. Oskowitz, A.; McFerrin, H.; Gutschow, M.; Carter, M. L.; Pochampally, R. Serum-deprived human multipotent mesenchymal stromal cells (MSCs) are highly angiogenic. Stem Cell Res. 6(3):215-225; 2011.

19. Parolini, O.; Alviano, F.; Bergwerf, I.; Boraschi, D.; De Bari, C.; De Waele, P.; Dominici, M.; Evangelista, M.;
Falk, W.; Hennerbichler, S.; Hess, D. C.; Lanzoni, G.; Liu, B.; Marongiu, F.; McGuckin, C.; Mohr, S.; Nolli, M. L.; Ofir, R.; Ponsaerts, P.; Romagnoli, L.; Solomon, A.; Soncini, M.; Strom, S.; Surbek, D.; Venkatachalam, S.; Wolbank, S.; Zeisberger, S.; Zeitlin, A.; Zisch, A.; Borlongan, C. V. Toward cell therapy using placenta-derived cells: Disease mechanisms, cell biology, preclinical studies, and regulatory aspects at the round table. Stem Cells Dev. 19(2):143- 154; 2010.

20. Payne, N. L.; Sun, G.; McDonald, C.; Layton, D.; Moussa, L.; Emerson-Webber, A.; Veron, N.; Siatskas, C.; Herszfeld, D.; Price, J.; Bernard, C. C. Distinct immunomodulatory and migratory mechanisms underpin the therapeutic potential of human mesenchymal stem cells in autoimmune demyelination. Cell Transplant. 22(8):1409-1425; 2013.

21. Praet, J.; Reekmans, K.; Lin, D.; De Vocht, N.; Bergwerf, I.; Tambuyzer, B.; Daans, J.; Hens, N.; Goossens, H.; Pauwels, P.; Berneman, Z.; Van der Linden, A.; Ponsaerts, P. Cell type-associated differences in migration, survival, and immunogenicity following grafting in CNS tissue. Cell Transplant. 21(9):1867-1881; 2012.

22. Praet, J.; Santermans, E.; Reekmans, K.; de Vocht, N.; Le Blon, D.; Hoornaert, C.; Daans, J.; Goossens, H.; Berneman, Z.; Hens, N.; Van der Linden, A.; Ponsaerts, P. Histological characterization and quantification of cellular events following neural and fibroblast(-like) stem cell grafting in healthy and demyelinated CNS tissue. Methods Mol. Biol. 1213: 265-283; 2014.

23. Reekmans, K.; De Vocht, N.; Praet, J.; Le Blon, D.; Hoornaert, C.; Daans, J.; Van der Linden, A.; Berneman, Z.; Ponsaerts, P. Quantitative evaluation of stem cell grafting in the central nervous system of mice by in vivo bioluminescence imaging and postmortem multicolor histological analysis. Methods Mol. Biol. 1052:125-141; 2013.

24. Reekmans, K.; Praet, J.; Daans, J.; Reumers, V.; Pauwels, P.; Van der Linden, A.; Berneman, Z. N.; Ponsaerts, P. Current challenges for the advancement of neural stem cell biology and transplantation research. Stem Cell Rev. 8(1):262-278; 2012.

25. Ronsyn, M. W.; Berneman, Z. N.; Van Tendeloo, V. F.; Jorens, P. G.; Ponsaerts, P. Can cell therapy heal a spinal cord injury? Spinal Cord 46(8):532-539; 2008.

26. Ronsyn, M. W.; Daans, J.; Spaepen, G.; Chatterjee, S.; Vermeulen, K.; D'Haese, P.; Van Tendeloo, V. F.; Van Marck, E.; Ysebaert, D.; Berneman, Z. N.; Jorens, P. G.; Ponsaerts, P. Plasmid-based genetic modification of human bone marrow-derived stromal cells: Analysis of cell survival and transgene expression after transplantation in rat spinal cord. BMC Biotechnol. 7:90; 2007.

27. Shinozuka, K.; Dailey, T.; Tajiri, N.; Ishikawa, H.; Kim, D. W.; Pabon, M.; Acosta, S.; Kaneko, Y.; Borlongan, C. V. Stem cells for neurovascular repair in stroke. J. Stem Cell Res. Ther. 4(4):12912; 2013.

28. Tambuyzer, B. R.; Bergwerf, I.; De Vocht, N.; Reekmans, K.; Daans, J.; Jorens, P. G.; Goossens, H.; Ysebaert, D. K.; Chatterjee, S.; Van Marck, E.; Berneman, Z. N.; Ponsaerts, P. Allogeneic stromal cell implantation in brain tissue leads to robust microglial activation. Immunol. Cell Biol. 87(4): 267-273; 2009.

29. Yu, S.; Tajiri, N.; Franzese, N.; Franzblau, M.; Bae, E.; Platt, S.; Kaneko, Y.; Borlongan, C. V. Stem cell-like dog placenta cells afford neuroprotection against ischemic stroke model via heat shock protein upregulation. PLoS One 8(9):e76329; 2013.

30. Zeger, S. L.; Liang, K. Y.; Albert, P. S. Models for longitudinal data - A generalized estimating equation approach. Biometrics 44(4):1049-1060; 1988. 